\title{
Smartphone hearing screening with integrated quality control and data management
}

\author{
De Wet Swanepoel*,†,\#, Hermanus C. Myburgh" $\$$, David M. Howe*,\$, Faheema Mahomed* \\ \& Robert H. Eikelboom $*,+$, \\ ${ }^{*}$ Department of Speech-Language Pathology and Audiology, University of Pretoria, Pretoria, South Africa, ${ }^{\dagger}$ Ear Sciences Centre, School of Surgery, \\ The University of Western Australia, Nedlands, Australia, ${ }^{\ddagger}$ Ear Science Institute Australia, Subiaco, Australia, ${ }^{\#}$ Callier Center for Communication \\ Disorders, University of Texas at Dallas, USA, and ${ }^{\$}$ Department of Electrical, Electronic and Computer Engineering, \\ University of Pretoria, Pretoria, South Africa
}

\begin{abstract}
Objective: To determine if a smartphone application could be used as a calibrated screening audiometer with real-time noise monitoring for school screening using automated test sequences. Design: The investigation comprised three studies. Study 1 evaluated calibration accuracy across four Samsung S5301 smartphones (Android v4.0.4) using commercial Sennheiser HD202 headphones. Study 2 involved referencing smartphone microphone sensitivity to narrowband noise intensity as measured in octave bands by a sound-level meter between 30 and $75 \mathrm{~dB}$ SPL (5 dB increments). Study 3 compared screening outcomes of smartphone based and conventional hearing screening. Study sample: Study 2: 15 normal-hearing subjects (age range, 18-22 years; all female). Study 3: 162 children (324 ears) aged 5 to 7 years. Results: Smartphone calibration at 20,30, and $40 \mathrm{~dB}$ was within $1 \mathrm{~dB}$ of recommended reference equivalent thresholds levels. Microphone calibration for noise monitoring had maximum variability across phones of $0.9,0.6$, and 2.9 dB at 1,2 , and $4 \mathrm{kHz}$, respectively, from reference intensities (30 to $75 \mathrm{~dB}$ SPL). Screening outcomes demonstrated no significant difference between smartphone and conventional audiometry with an overall referral rate of $4.3 \%$ and $3.7 \%$, respectively. Conclusions: The newly developed smartphone application can be accurately calibrated for audiometry with valid real-time noise monitoring, and clinical results are comparable to conventional screening.
\end{abstract}

Key Words: Ambient noise; audiometry; calibration; childhood hearing loss; hearing screening; pure-tone audiometry; school screening

Hearing loss is a significant global health-care burden because of its widespread prevalence and long-term consequences for affected individuals, communities and society. Recent estimates by the World Health Organization indicate that 360 million persons, comprising $5.3 \%$ of the global population, suffer from permanent disabling hearing loss (WHO, 2013a). More than 32 million of these are children, of whom the vast majority will not have access to early detection services for their hearing loss (WHO, 2010, 2013a).

Children with hearing loss are at risk for delayed speech, language, and cognitive skills, which translates to reduced literacy, academic and socio-emotional development, and higher risks of failure and fall-out in schools (WHO, 2013a). Unidentified childhood hearing loss has historically been shown to dramatically deteriorate educational achievement and ultimately vocational outcomes (AAA, 2011;
Holden-Pitt \& Diaz, 1998). Even minimal and unilateral permanent hearing losses may result in poorer educational test performance, higher incidence of failed grades, and greater dysfunction in areas such as behavior, energy, stress, social support, self-esteem, and socio-emotional aspects (Tharpe \& Bess, 1991; Bess et al, 1998; McKay et al, 2008).

Early detection of childhood hearing loss provides the opportunity to forestall these negative consequences. Unfortunately in underserved regions such as sub-Saharan Africa, where an estimated 6.8 million children $(<15$ years of age) suffer from disabling hearing loss, access to ear and hearing related services such as hospital- or school-based screening is severely limited (Fagan \& Jacobs, 2009; Goulios \& Patuzzi, 2008; WHO, 2013b). This is primarily due to the shortage of hearing health-care professionals, with regions 


\begin{tabular}{ll}
\multicolumn{2}{l}{ Abbreviations } \\
FFT & Fast Fourier transform \\
MPANL & Maximum permissible ambient noise level \\
NBN & Narrowband noise \\
RETSPL & Reference equivalent threshold sound pressure level \\
SLM & Sound level meter
\end{tabular}

such as sub-Saharan Africa and South-East Asia typically presenting with less than one audiologist for every million people (Fagan \& Jacobs, 2009; WHO 2013b). Secondary to human resources, the expense of audiological equipment for hearing assessment severely restricts access to services (Swanepoel et al, 2010a). As a result there has been growing interest in recent years to capitalize on the increasingly widespread availability of global connectivity (Kelly \& Minges, 2012; Internet World Stats, 2014; Clark \& Swanepoel, 2014) to make services more accessible and to utilize novel approaches incorporating automation alongside more cost-effective audiological equipment solutions such as mobile phones (Clark \& Swanepoel, 2014; Swanepoel et al, 2010a, 2010b; Szudek et al, 2012).

Utilization of telehealth approaches for hearing assessment has steadily gained acceptance in recent years (Swanepoel \& Hall, 2010; Swanepoel et al, 2010a). More recently mobile health (mHealth), often seen as a sub-set of eHealth, has also emerged as a possible means of hearing assessment (Clark \& Swanepoel, 2014). This area is particularly attractive because of the widespread penetration of mobile phones and cellular network reception globally even in underserved world regions such as sub-Saharan Africa (Kelly \& Minges, 2012).

In hearing health care, there are already a number of smartphone applications available to conduct basic hearing assessments (e.g. pure-tone audiometry, speech audiometry). One such application developed for Apple's iOS devices (iPhone \& iPad) called uHear ${ }^{\mathrm{TM}}$ (Unitron) provides a self-directed assessment of air conduction hearing thresholds. Three published studies have compared the performance of this application for hearing assessment in different populations (Handzel et al, 2013; Khoza-Shangase \& Kassner, 2013; Szudek et al, 2012). In a group of adults Szudek et al (2012) demonstrated reasonably accurate results for ruling out moderate hearing loss and to quantify a general degree of hearing loss. In normal-hearing persons, however, it proved to be inaccurate with overestimation of thresholds. A study on school children between 8 and 10 years of age using the $\mathrm{uHear}^{\mathrm{TM}}$ application showed inaccurate threshold results that were significantly elevated (Khoza-Shangase \& Kassner, 2013). A clinical study of patients with unilateral sudden onset hearing loss demonstrated that the $\mathrm{uHear}^{\mathrm{TM}}$ application was useful to categorize the type of loss during otological consultations with a sensitivity of $76 \%$ and specificity of $91 \%$ (Handzel et al, 2013). Two other iOS applications using Apple products (iPhone, iPod, or iPad) for air conduction threshold audiometry were recently evaluated by comparison to conventional audiometry (Kam et al, 2012; Foulad et al, 2013). Results of these self-administered tests demonstrate close threshold correspondence to conventional air conduction audiometry.

For screening purposes hearing threshold determination, as reported by the studies that investigated iOS applications (KhozaShangase \& Kassner, 2013; Szudek et al, 2012; Kam et al, 2012; Foulad et al, 2013), is inappropriate since a screen constitutes a specified screening intensity level (e.g. $20 \mathrm{~dB}$ HL) across selected frequencies (e.g. 1, 2, and $4 \mathrm{kHz}$ ) denoting only pass or fail for patients. True hearing screening applications have been reported on personal computers and tablets (McPherson et al, 2010; Kam et al, 2013). Initial findings did however reveal poor test performance with excessive referral rates which were attributed to factors such as ambient noise influences, lack of a conditioning stimulus prior to commencing the screen, and a possible order effect favoring conventional screening (McPherson et al, 2010; Kam et al, 2013). A recent follow-up study using the tablet-based (Android) technology, which employed an automated multimedia platform operated by the child with noise cancellation headphones, was conducted on a large sample of preschool children and demonstrated a reduced referral rate of $9.3 \%$ at a screening level of $30 \mathrm{~dB}$ at 1,2 , and $4 \mathrm{kHz}$ (Wu et al, 2014).

Whilst demonstrating real promise, particularly the applications reported by Kam et al (2012) and Foulad et al (2013), there are still important challenges that remain before smartphones could be used as legitimate audiometric devices. Limitations include the fact that all current studies have been conducted using iOS-based devices with intraconchal headphones supplied with the devices and for which there is no standardized calibration procedure as yet (Handzel et al, 2013; Khoza-Shangase \& Kassner, 2013; Szudek et al, 2012). As a result standardized acoustic calibration of the device and headphones to verify compliance to current standards for audiometric equipment is not available. Other studies utilized non-audiometric headphones for which there also is no standardized reference equivalent threshold sound pressure levels (RETSPLs) specified (Kam et al, 2013; $\mathrm{Wu}$ et al, 2014). Additionally, current applications do not offer a real-time quality control facility for environmental noise influences. Another important clinical limitation related specifically to the use of iOS-based devices pertains to the fact that they are premium products that are often too expensive for underserved areas such as subSaharan Africa where the need for services is typically greatest.

Towards finding a cost-effective hearing screening solution the current study investigated the development and validation of a smartphone application (hearScreen ${ }^{\mathrm{TM}}$ ) utilizing an inexpensive smartphone (Android OS) and headphone for hearing screening with automated test sequences employing real-time monitoring of environmental noise and data management facilities. The hearScreen ${ }^{\mathrm{TM}}$ application was developed at the University of Pretoria using the Android SDK (software development kit) version 21.0.1 via the Eclipse IDE (integrated development environment) version 4.2.1.

Study aims were to (1) determine the validity of smartphone acoustic calibration using non-audiometric headphones; (2) determine the validity of smartphone-based environmental noise monitoring; and (3) conduct a preliminary clinical validation in a sample of school children.

\section{Study 1: Validity of smartphone acoustic calibration}

\section{Methods}

At present audiometric calibration standards are only available for specific audiometric headphones (e.g. TDH39 and HDA200 headphones) for which reference equivalent threshold sound pressure levels (RETSPLs) are specified (ISO 389-1, 1998; ISO 389-8, 2004; ANSI/ASA S3.6, 2010). For this study a headphone not normally used for audiometry was employed. The Sennheiser HD202 II (Sennheiser, Wedemark, Germany) supra-aural headphone was selected because it is an inexpensive (US\$35 list price), internationally available headphone with a wide frequency response and reasonable attenuation. Since the completion of the current study 
an investigation has been initiated to establish RETSPLs for this headphone according to ISO 389-9 (2009).

The headphone was connected using a $3.5 \mathrm{~mm}$ stereo plug to a Samsung (Daegu, South Korea) Galaxy Pocket Plus S5301 smartphone (US\$80 list price) running the hearScreen ${ }^{\mathrm{TM}}$ application on Android OS version 4.0.4 (Google, Mountain View, USA). The application generates pure-tone signals with a calibration function to approximate the required RETSPL specified for standard supraaural audiometric headphones (TDH39). For this study pure tone signals were evaluated at 1,2 and $4 \mathrm{kHz}$ and at intensities of 20, 30 and $40 \mathrm{~dB}$ HL as currently recommended frequency and intensity ranges for childhood hearing screening. (ASHA, 1997; AAA, 2011; Skarzynski \& Piotrowska, 2012).

The output of the Sennheiser HD202 headphone in this study was measured with a standard artificial ear Brüel \& Kjær Type 4152 coupler (Brüel \& Kjær, Nærum, Denmark). The headphone was connected to the coupler with a force of $3( \pm 0.5)$ Newton, which was the typical force of the headband when headphones were $145 \mathrm{~mm}$ apart while the midpoint of the headband was $129 \mathrm{~mm}$ above an imaginary line between the two headphones as specified (IEC 60645-1, 2012; ISO 389-9, 2009). The coupler was connected to a Rion NA-28, Integrating Sound Level Meter and 1/3 Octave Band Analyser (Rion, Tokyo, Japan), which measured the output of the headphones. All measurements were made in a double-walled sound booth certified for free-field testing.

The right and left outputs were measured separately. At each frequency tested, the signal amplitude on the phone was adjusted according to the readings from the sound level meter (SLM) to ensure that all of the pre-specified output levels (20, 30, and 40 $\mathrm{dB} \mathrm{HL}$ ) measured in $\mathrm{dB}$ SPL were equivalent to the $\mathrm{dB} \mathrm{HL}$ values set in the system, with the conversion from the artificial ear to TDH39 headphones (Telephonics, Farmingdale, USA) according to current standards (ISO 389-1, 1998) applied. This was repeated with four mobile devices with the same headphone to determine if the phone and headphone combinations could be calibrated to within the required range of the target RETSPL levels. In addition to this, the output of two phones were verified with three batteries inserted each with power levels of $<10 \%, 50 \%$, and $>90 \%$ to assess if this had any influence on the sound output.

\section{Results and Discussion}

The maximum difference across the calibrated intensity levels for the four phones compared to the required calibration intensity (ISO
389-1, 1998) was less than $1 \mathrm{~dB}$ across all intensity and frequency levels (Table 1). According to audiometric calibration standards (ISO 389-1, 1998) air conduction pure-tone signals $(1,2$, and $4 \mathrm{kHz})$ are considered to meet RETSPLs if they are within $\pm 3 \mathrm{~dB}$. This means all pure-tone signals generated by the application across four smartphones for the left and right headphone could be acoustically calibrated. Calibration was performed independently at each intensity level for each frequency. The application stores the corresponding smartphone intensity setting to correspond to the specific $\mathrm{dB}$ HL intensity referenced to the RETSPL. In this way every frequency and intensity setting can be calibrated according to the prescribed RETSPL's. Different battery power levels $(<10 \%, 50 \%$, and $>90 \%$ ) did not have any influence on pure-tone signal output on the smartphones.

As in previous reports on smartphone-based audiometry (Handzel et al, 2013; Khoza-Shangase \& Kassner, 2013; Szudek et al, 2012; Foulad et al, 2013; Kam et al, 2012) the headphones utilized in this study were non-audiometric headphones. As opposed to the intraconchal (iOS device) headsets of previous studies the headphones in the current study were, however, supra-aural headphones with closer correspondence to the typical audiometric TDH39 supraaural headphones. As a result the RETSPLs specified for the TDH39 supra-aural audiometric headphone was used for calibration whilst taking into consideration the coupling force difference between the headbands (3 compared to 4.5 Newton for the Sennheiser HD202 and TDH39 headphones) as specified when determining RETSPLs for non-audiometric headphones (ANSI/ASA S3.6-2010; ISO 389-9, 2009). Ideally, headphone specific RETSPLs should be established for non-standard audiometric headphones according to prescribed methods to take into account the acoustic impedance difference between headphones, other than the headband force which was accounted for in this study (ISO 389-9, 2009). A follow-up study is underway to standardize the Sennheiser HD202 supra-aural headphones for audiometric screening purposes by determining RETSPL values according to ISO 389-9 (2009).

Study 1 demonstrates that accurate acoustical calibration can be attained using the hearScreen ${ }^{\mathrm{TM}}$ application on a low-cost smartphone (Android OS). Furthermore it provides the first evidence of smartphone-based audiometry on devices other than iOS products and is the only application to date that allows for unique calibration of phone and headphone pairs according to prescribed standards (ANSI/ASA S3.6-2010; ISO 389-1, 1998). This opens up opportunities for low-cost hearing screening solutions whereby

Table 1. Smartphone acoustic calibration correspondence to reference equivalent threshold sound pressure levels (RETSPLs) at different intensities (20, 30, and 40 dB HL) using Sennheiser HD202 supraaural headphones. Four smartphones (Samsung Galaxy Pocket Plus S5301) were calibrated at three intensity levels (20, 30, and $40 \mathrm{~dB}$ HL) using the same headphones according to RETSPLs for TDH39 supra-aural headphones (ISO 389-1:1998; ANSI/ASA S3.6:2010). Eight measurements (four in the left and four in the right) were made to approximate the RETSPL levels at every intensity for each smartphone.

\begin{tabular}{|c|c|c|c|c|c|c|c|c|c|}
\hline & \multicolumn{9}{|c|}{ Calibration levels } \\
\hline & \multicolumn{3}{|c|}{$20 \mathrm{dBH} H$} & \multicolumn{3}{|c|}{$30 \mathrm{~dB} H L$} & \multicolumn{3}{|c|}{$40 \mathrm{dBHL}$} \\
\hline & $1 \mathrm{kHz}$ & $2 \mathrm{kHz}$ & $4 \mathrm{kHz}$ & $1 \mathrm{kHz}$ & $2 \mathrm{kHz}$ & $4 \mathrm{kHz}$ & $1 \mathrm{kHz}$ & $2 \mathrm{kHz}$ & $4 \mathrm{kHz}$ \\
\hline Average difference* & 0.9 & 0.5 & -0.6 & -0.7 & -0.7 & -0.4 & -0.5 & -0.6 & -0.1 \\
\hline $\mathrm{SD}$ & 0.1 & 0.2 & 0.2 & 0.2 & 0.2 & 0.2 & 0.2 & 0.2 & 0.3 \\
\hline Maximum difference (abs) & 1.0 & 0.8 & 0.8 & 1 & 0.9 & 0.7 & 0.8 & 0.8 & 0.4 \\
\hline
\end{tabular}

*Average difference between smartphone calibration intensity and specified RETSPL for TDH39 supra-aural headphones $(n=8)$. 
smartphones can be utilized as screening audiometers with headphones that can be calibrated according to prescribed standards.

\section{Study 2: Validity of smartphone environmental noise monitoring}

Study 2 included two phases. The first phase involved calibration of the smartphone microphone in reference to a Type 1 SLM for noise monitoring. The second phase determined attenuation of the smartphone screening headphones compared to standardized headphones to specify maximum permissible ambient noise levels (MPANLs) for the smartphone-based environmental noise-monitoring feature.

\section{Methods \\ PHASE 1}

For phase one the microphones of five Samsung Galaxy Pocket Plus S5301 smartphones were assessed to determine reference intensity levels corresponding to the intensity recorded by a Type 1 SLM (Rion NA-28) with a free-field 1/2-inch microphone (UC-59). A free field setup was utilized with narrow-band noise $(\mathrm{NBN})$ presented through speakers at $5-\mathrm{dB}$ increments from 30 to $75 \mathrm{~dB}$ SPL at 1 , 2 , and $4 \mathrm{kHz}$. This was achieved by setting up a Type 1 SLM in a double-walled soundproof booth $\left(2.5 \times 2.5 \mathrm{~m}^{2}\right)$ at $0^{\circ}$ azimuth, $0.5 \mathrm{~m}$ from the sound field speaker (midpoint $87.5 \mathrm{~cm}$ above the floor) using a slow integration of 1 second (Figure 1). The NBN intensity was adjusted to the required SLM intensity (30 dB SPL) using 1-dB

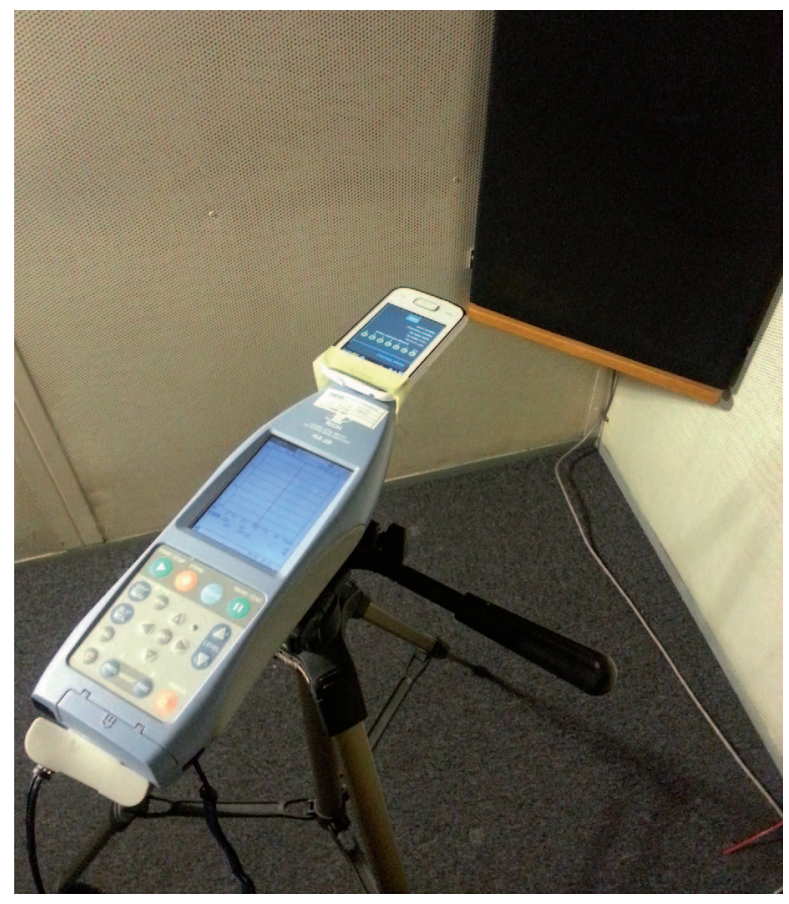

Figure 1. Test setup for determining signal intensity on the smartphone microphone compared to a Type 1 sound level meter (SLM). Smartphone and SLM microphones similar position and distance from the free-field speaker $(1 \mathrm{~cm}$ vertical distance from each other) measuring continuous narrowband noise. SLM provided dB SPL reference for corresponding smartphone averaged FFT integral (16 kHz sampling rate with 500 512-point FFTs). increments on the audiometer. Subsequently the NBN was continuously presented from 30 to $70 \mathrm{~dB}$ SPL in $5 \mathrm{~dB}$ increments (ANSI 3-1, 1999 (R2008)). At each intensity increment the corresponding averaged fast Fourier transform (FFT) integral measured from the smartphone microphone was recorded. A sampling rate of $16 \mathrm{kHz}$ was used to acquire the signal on the smartphone with 500512 point FFTs constituting the averaged FFT value. Measurements were made on the SLM and smartphone in octave bandwidths of $1 \mathrm{kHz}$ $(0.71-1.42 \mathrm{kHz}), 2 \mathrm{kHz}(1.42-2.84 \mathrm{kHz})$, and $4 \mathrm{kHz}(2.84-5.68$ $\mathrm{kHz}$ ). At each NBN intensity increment per frequency, as referenced to the SLM, the results of 500 FFT of 512 blocks each were averaged on the smartphone to produce a reference value.

The smartphone amplitude recorded at each intensity increment (30 to $70 \mathrm{~dB}$ in 5-dB steps) was compared and averaged at 1,2, and $4 \mathrm{kHz}$ octave bands across the five phones referenced to the NBN intensity levels at 30 to $75 \mathrm{~dB}$ SPL (in 5-dB increments) as measured by the type 1 SLM. Averaged values were subsequently interpolated, using spline interpolation, to produce a mapping between the FFT averaging outputs and the ambient noise levels.

\section{Phase 2}

Phase two recruited a sample of 15 normal-hearing subjects (age range, 18-22 years; all female) in order to determine the attenuation of various headphones. The experimental setup was according to the procedures described in ISO 8253-1 (2010) for determining maximum permissible ambient noise levels for non-standard supraaural headphones. The procedure involved determination of puretone thresholds with a Type 1 GSI 61 audiometer (Grayson Stadler, Eden Prairie, USA) at octave and inter-octave frequencies from 0.25 to $8 \mathrm{kHz}$ in the free field within a double-walled sound booth. Four free-field audiograms were conducted in a counter-balanced order to measure headphone attenuation. These conditions were (1) free field thresholds without any headphones in place; (2) ER-3A (Etymyotic Research, Elk Grove Village, USA) insert earphones in place bilaterally; (3) TDH-39 supra-aural headphones; (4) Sennheiser HD202 supra-aural headphones. The level of attenuation provided by the various headphones was calculated as the difference in threshold at individual frequencies with and without headphones fitted.

The azimuth of the free field speakers was 45 degrees on the left and right, 1 meter from the subject's ears. Pure tones were presented from both speakers simultaneously; therefore results were not earspecific and represented the best hearing thresholds, irrespective of ear. Subjects were instructed to respond to stimuli by pressing the audiometer response button. A modified Hughson-Westlake threshold seeking method was used to determine hearing thresholds starting at $1 \mathrm{kHz}$ and $30 \mathrm{~dB} \mathrm{HL}$, moving on to lower octave frequencies of 0.25 and $0.5 \mathrm{kHz}$, and then higher octave frequencies from 2 to 8 $\mathrm{kHz}$ (including 3 and $6 \mathrm{kHz}$ ). Hearing thresholds were $20 \mathrm{~dB}$ or less in all cases (except for one threshold of $25 \mathrm{~dB}$ at $8 \mathrm{kHz}$ ).

Analysis included the mean and standard deviation of attenuation across frequencies and a Wilcoxon signed ranks test to assess differences across headphones ( $\mathrm{p}<0.05$ indicated significant difference between headphones).

\section{Results and Discussion}

The averaged FFT integrals of the noise measured by the hearScreen ${ }^{\mathrm{TM}}$ software across five smartphones encompassed a range of 0.3 to 309 across the test frequencies and intensities from 30 to $75 \mathrm{~dB}$ (Figure 2). An interpolation of averaged FFT integral values allowed for the determination of values at intermediate intensities 


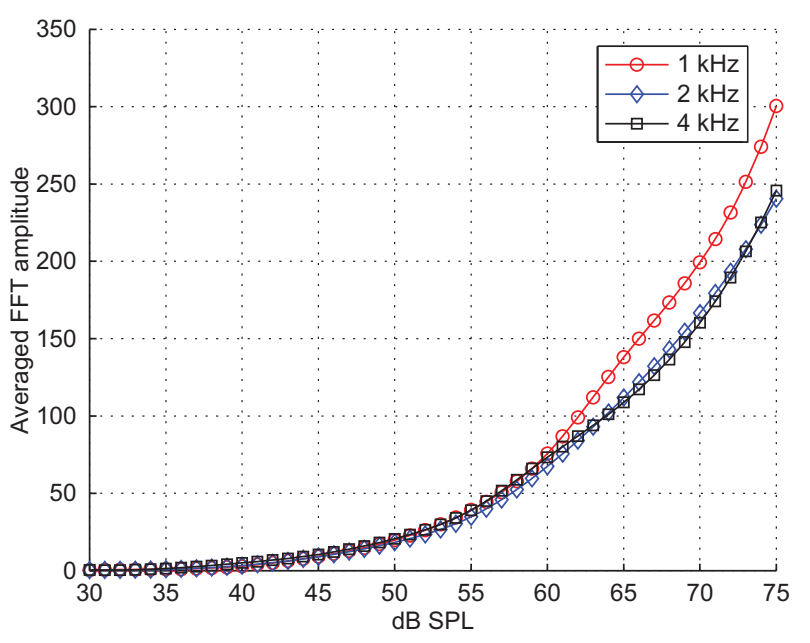

Figure 2. Average smartphone microphone $(n=5)$ sensitivity in $\mathrm{dB}$ SPL interpolated from discreet reference increments of $5 \mathrm{~dB}$ between 30 and $75 \mathrm{~dB}$ SPL, as measured by a type 1 SLM using narrow-band noise.

and provided a function on the smartphone for noise measurements between 30 and $75 \mathrm{~dB}$ SPL. Maximum variability from the reference intensities ( 30 to $75 \mathrm{~dB}$ SPL as measured by the SLM) across the five smartphones was $0.9,0.6$, and $2.9 \mathrm{~dB}$ at 1,2 , and $4 \mathrm{kHz}$ respectively (Figure 3).

Average attenuation of the Sennheiser HD202 headphones only differed significantly from supra-aural TDH39 headphones at $0.25 \mathrm{kHz}$ where it was significantly poorer, and at $8 \mathrm{kHz}$ where it was significantly better $(\mathrm{p}<0.05)$. Insert earphone attenuation was significantly higher $(\mathrm{p}<0.05)$ than Sennheiser HD202 headphones across all frequencies assessed, except at 2, 4, and $6 \mathrm{kHz}$ (Table 2). The MPANLs for audiometric testing using the Sennheiser HD202 headphones were therefore similar to that prescribed for TDH39 headphones (ANSI/ASA S3.1-1999(R2013)).

Employing a screening level of $20 \mathrm{~dB}$ HL using the Sennheiser HD202 headphone therefore requires MPANLs across octave bands for 1,2 , and $4 \mathrm{kHz}$ to be 43.7, 52.0, and 55.6 dB SPL respectively (ANSI/ASA S3.1-1999(R2013)). The noise-monitoring feature of the smartphone was subsequently programmed to utilize the headphone-specific MPANL to monitor environmental noise in real-time

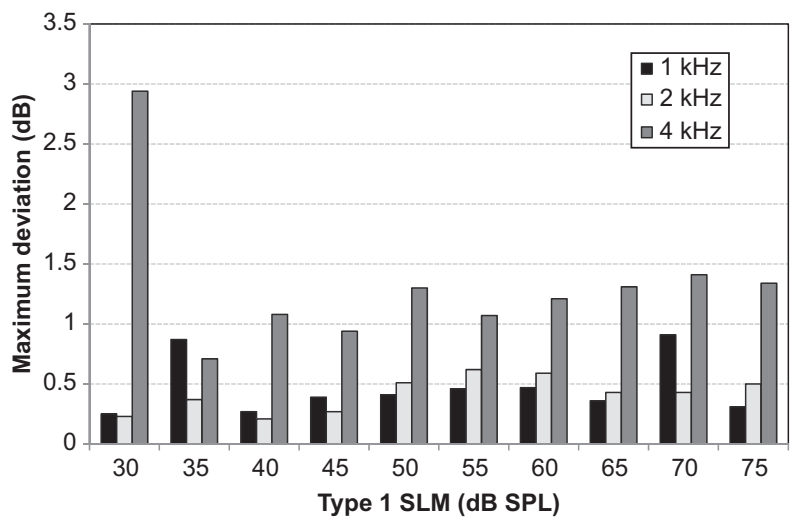

Figure 3. Maximum deviation in intensity measurements across five smartphone microphones compared to reference sound intensities (dB SPL). updates of $32 \mathrm{~ms}$. As illustrated in Figure 4 the noise monitoring on the smartphone was scaled according to the MPANL at the specific intensity and frequency being tested to provide the operator with an indication of the environmental compliance.

Noise interference is recognized as one of the most important contributors to over-referrals and the subsequent inefficiency of hearing screening programs for children (Bamford et al, 2007; FitzZaland \& Zink, 1984; AAA, 2011; ASHA, 1997; Lo \& McPherson, 2013). Current recommendations to ensure compliant noise levels include determining ambient noise levels in the test environment prior to testing, using a sound level meter (AAA, 2011; ASHA, 1997). The problem with this method is that many school health services do not have sound level meters (AAA, 2011) and even if they do, noise levels in schools can change significantly throughout the day, making initial measurements obsolete. An alternative approach is to use biologic noise level checks prior to hearing screening. This involves establishing thresholds at least $10 \mathrm{~dB}$ lower than the screening level at all frequencies on a person with known normal hearing. However, apart from being subjective this method suffers the same caveat of the primary method in that noise levels do not remain consistent throughout the day.

The ability to provide noise monitoring referenced to MPANLs during testing allows screening operators the unique possibility to have real-time feedback on the ambient noise levels to ensure compliance. To position the microphone optimally during operation the application requires the phone to be held upside-down so that the microphone faces upwards, pointing towards the subject being tested (Figure 5). The accuracy of the monitoring is sufficient for valid indications of whether noise levels exceed MPANLs (within $1 \mathrm{~dB}$ for 1 and $2 \mathrm{kHz}$; within $1.5 \mathrm{~dB}$ for $4 \mathrm{kHz}$ except at 30 $\mathrm{dB}$ SPL which was within $<3 \mathrm{~dB}$ ). For screening levels between 20 and $40 \mathrm{~dB}$ HL the smartphone microphones demonstrate minimal variability $(<0.5 \mathrm{~dB}$ at $1 \mathrm{kHz} ;<0.6 \mathrm{~dB}$ at $2 \mathrm{kHz}$; and $<1.4 \mathrm{~dB}$ at $4 \mathrm{kHz}$ ) that falls within the $1.5 \mathrm{~dB}$ tolerance specified for Type 1 SLM's when measuring broadband noise (ANSI S1.4:1983 (R2007)). (ANSI, 1983(R2007)). A recent evaluation of smartphone-based SLM applications also confirmed the accuracy of certain types of applications for noise measurements (Kardous \& Shaw, 2014).

The average noise level during the time each frequency in each ear is assessed is stored in the internal device datasheet for quality control of screenings conducted.

\section{Study 3: Clinical validity of smartphone-based audiometry}

Study 3 piloted the hearScreen ${ }^{\mathrm{TM}}$ smartphone on 162 school children between 5.6 and 7.7 years of age ( 6.5 mean \pm 0.4 SD) against conventional audiometry screening. Ethical clearance was obtained from the University of Pretoria Institutional Review Board before data collection commenced.

\section{Methods}

Screening procedures were conducted in a counterbalanced sequence (alternating between starting with conventional or smartphone-based screening) following the same protocol. The screening intensity level was $25 \mathrm{~dB}$ HL at 1,2, and $4 \mathrm{kHz}$. Left ears were tested first with an initial presentation at $1 \mathrm{kHz}, 10 \mathrm{~dB}$ above the screening level as a conditioning presentation, followed by the screening at 1,2 , and $4 \mathrm{kHz}$. If a child did not respond at a specific intensity it was repeated once. Referral at any frequency in an ear constituted an 
Table 2. Average attenuation (dB) provided by standard audiometric headphones (TDH39 and insert earphones) compared to Sennheiser HD202 headphones $(n=15)$.

\begin{tabular}{|c|c|c|c|c|c|c|c|c|}
\hline & \multicolumn{8}{|c|}{ Average attenuation $(S D)$ across frequency $(\mathrm{kHz})$} \\
\hline & 0.25 & 0.5 & 1 & 2 & 3 & 4 & 6 & 8 \\
\hline TDH39 & $3.0^{*}(5.3)$ & $6.3(6.9)$ & $11.0(8.9)$ & $17.0(7.5)$ & $24.3(10)$ & $29.7(9.2)$ & $23.7(10.9)$ & $14.0^{*}(10.2)$ \\
\hline Insert earphones & $12.0^{*}(9.6)$ & $16.0 *(10.0)$ & $18.3^{*}(10.3)$ & $21.7(10.3)$ & $31.0^{*}(11.4)$ & $31.0(7.4)$ & $32.0(7.5)$ & $29.7^{*}(10.8)$ \\
\hline Sennheiser HD202 & $0.3(3.5)$ & $4.0(3.4)$ & $10.7(4.2)$ & $15.0(7.8)$ & $22.0(7.5)$ & $28.3(7.7)$ & $28.3(13.2)$ & $24.3(7.8)$ \\
\hline
\end{tabular}

*Significant difference from HD202 headphone attenuation $(\mathrm{p}<0.05$; Wilcoxon signed ranks test).

initial referral. A rescreen was done immediately following a refer result using the same screening audiometer on which the initial refer was recorded.

Screening was conducted by audiology students from the University of Pretoria in a quiet room provided by a local school where testing was conducted. All pupils in reception grade and grade 1, for whom parents provided informed consent, participated. Conventional screening was conducted with one of two screening audiometers, a GSI Auto Tymp (Grayson Stadler, Eden Prairie, USA) and an Interacoustics Impedance Audiometer AT 235 (William Demant, Smørum, Denmark), both using Telephonics TDH 39P headphones. Smartphone-based screening was conducted using two Samsung Galaxy Pocket Plus S5301 phones running the hearScreen ${ }^{\mathrm{TM}}$ Android OS application with Sennheiser HD202 supra-aural headphones. An audiologist trained screeners to conduct both conventional and hearScreen ${ }^{\mathrm{TM}}$ screening audiometry. The same audiologist also monitored all screening during onsite testing. Both the conventional and smartphone-based audiometers were calibrated using the calibration setup described in study 1 before any clinical data was collected.

For both conventional and smartphone-based screening, children were instructed to raise their hand if they heard the sound. The screener sat behind the children (Figure 5). Smartphone-based screening was conducted using the hearScreen ${ }^{\mathrm{TM}}$ application, which employs the specified screening protocol in an automated manner (Figure 4). The screener (device operator) presses the 'present' icon to have the pure-tone signal presented. Subsequent to the presentation the screener can either indicate whether the child heard the signal or not (YES/NO). There is also a 'replay' icon in cases where this may be required (allowed once only per test frequency). Based on the response selected the screening protocol adjusts to the next intensity and frequency selection.

Smartphones were connected to a $3 \mathrm{G}$ cellular network through which the smartphone-based screening results were emailed to one of the researchers after each screening session conducted over the four days of data collection (Figure 6). The data was transmitted as an MS Excel datasheet that included the demographic data, screening results for each frequency per ear, overall screen result per ear and per subject, test time, and the average noise levels per octave band at the screening frequency (per ear). The recorded noise level consists of the averaged ambient noise recorded by the smartphone during the pure-tone presentation (1.2 seconds) in the octave band corresponding to the test frequency.

Data analyses included a cross-tabulation of test outcomes with a McNemar's test applied to assess the significance of the difference between screening test outcomes $(\mathrm{p}<0.05$ was considered significant). The paired samples T-test was used to determine if there was a significant difference between test times for the two screening tests $(\mathrm{p}<0.05$ was considered significant $)$

\section{Results and Discussion}

Smartphone and conventional hearing screening methods were in agreement in $97.8 \%$ of ears assessed (Table 3). In $0.9 \%$ of cases, however, the mobile phone screening passed an ear whilst the

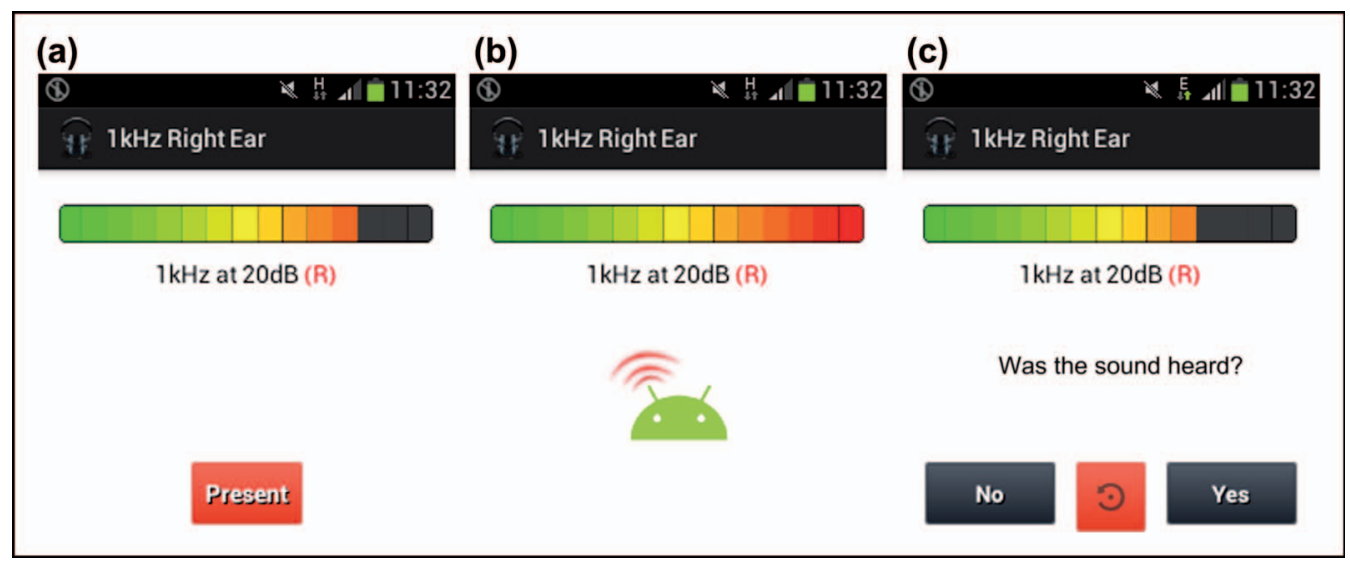

Figure 4. Smartphone-based audiometry (hearScreen ${ }^{\mathrm{TM}}$ ) screening interface. (a) Presentation screen with noise monitoring bar on top panel. The environmental noise exceeds the maximum permissible ambient noise levels when red (dark grey) bar against right limit. (b) Presentation of pure-tone signal. For illustration purposes this screenshot indicates environmental noise exceeding permissible levels during signal presentation. (c) After signal presentation the operator indicates whether the child responded or not. Depending on the response the protocol automatically moves to the next protocol step. A button (blue for left, red for right) between the 'No' and 'Yes' buttons allow for repeating the presentation of the signal once, before indicating a response. 


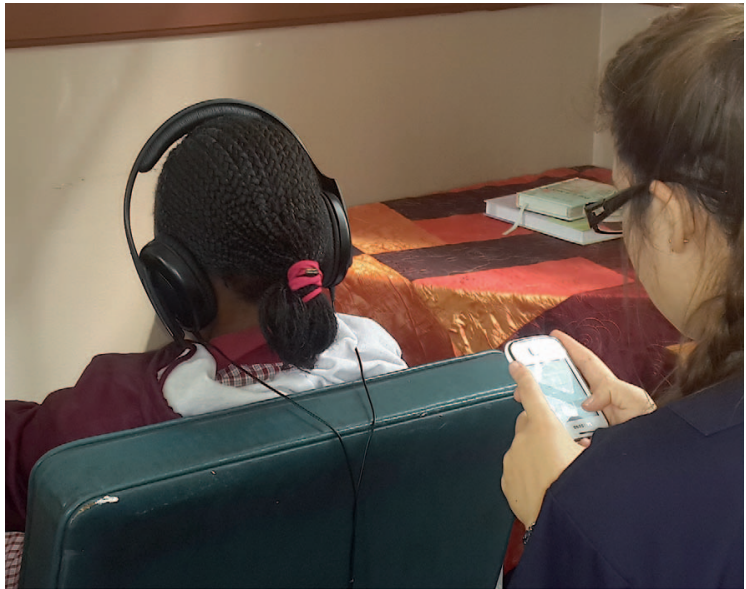

Figure 5. Clinical hearing screening test on school child using smartphone with hearScreen ${ }^{\mathrm{TM}}$ application and HD202 headphones. Phone is held upside-down to ensure the microphone faces towards the test subject for environmental noise monitoring.

manual screening referred the ear, and in $1.2 \%$ of cases manual screening passed an ear whilst the mobile phone screening referred the ear (Table 3). There was no statistically significant difference between the test results for conventional and smartphone-based hearing screening $(p>0.05)$. In terms of referred children (based on a referral in either ear) smartphone based audiometry had a $4.3 \%$ referral rate $(7 / 162$ children) compared to $3.7 \%$ for conventional audiometry (6/162 children). Four of the seven children referred on smartphone screening also referred on conventional screening. Diagnostic follow-up results were not available during this preliminary data collection phase, which meant true sensitivity and specificity rates could not be determined against a diagnostic gold standard.

Referral rates of a tablet-based screening system recently reported by $\mathrm{Wu}$ et al (2014) were $6.5 \%$ and $4 \%$ for 5 and 6 year old children respectively using a screening intensity $5 \mathrm{~dB}$ higher than the current study (30 dB HL). The hearScreen ${ }^{\mathrm{TM}}$ smartphone referral rate was similar $(4.3 \%)$ for children aged 5 to 7 , but employed a screening intensity of $25 \mathrm{~dB}$ HL. If screening was conducted at $30 \mathrm{~dB}$ HL the referral rate is expected to be lower.

Average smartphone screening duration was 60.9 seconds (38.6 $\mathrm{SD}$; range 19-325) compared to 67.1 seconds (47.1 SD; range 21-350) for conventional screening audiometry. Although smartphone screening was shorter in duration by an average of 6.2 seconds
Table 3. Cross tabulation of screening outcomes for ears using conventional and mobile phone based hearing screening $(n=324$ ears).

\begin{tabular}{llccc}
\hline & \multicolumn{3}{c}{ Conventional screening } \\
\cline { 2 - 5 } & \multicolumn{2}{c}{ Pass } & Refer & Total \\
\hline Mobile phone screening & Pass & $96.3 \%(312)$ & $0.9 \%(3)$ & $\mathbf{9 7 . 2 \% ( 3 1 5 )}$ \\
& Refer & $1.2 \%(4)$ & $1.5 \%(5)$ & $\mathbf{2 . 7 \% ( 9 )}$ \\
& Total & $\mathbf{9 7 . 5 \% ( 3 1 6 )}$ & $\mathbf{2 . 4 \% ( 8 )}$ \\
\hline
\end{tabular}

(57.5 SD), compared to conventional screening this difference was not significant $(\mathrm{p}>0.05)$. The average test time reported by $\mathrm{Wu}$ et al (2014) was 2.49 minutes compared to 1 minute (60.9 seconds) for the hearScreen ${ }^{\mathrm{TM}}$ smartphone test.

Average noise levels, as recorded with the smartphones, during testing was 39.0 (4.8 SD), 38.7 (4.8 SD), and 38.2 (5.1 SD) dB SPL for octave bands 1,2 , and $4 \mathrm{kHz}$ respectively, with maximum noise levels at 50, 55, and $53 \mathrm{~dB}$ SPL for 1,2 , and $4 \mathrm{kHz}$ respectively. The MPANL's per octave band for screening at $25 \mathrm{~dB}$ considering the slight attenuation difference between the Sennheiser HD 202 headphones used in this study and those specified for the TDH39 supraaural headphones (ANSI/ASA S3.1-1999(R2013)) was 49, 57, and 61 $\mathrm{dB}$ SPL for 1,2 , and $4 \mathrm{kHz}$ respectively. Only one screening at $1 \mathrm{kHz}$ was presented during noise (50 dB SPL) that exceeded the MPANL (by $1 \mathrm{~dB}$ ). In this case however the child still responded to the signal. The software is programmed to provide a pop-up noise warning followed by a retest at that frequency when noise exceeds the MPANL, and no response was elicited from the child in that instance.

Clinical screening outcomes using hearScreen ${ }^{\mathrm{TM}}$ smartphone and conventional audiometry demonstrate comparable referral rates and average test times with smartphone advantages in terms of being lightweight, portable, monitoring environmental noise, and offering data capturing, sharing and management functions.

\section{Limitations}

Limitations to the study include the fact that audiology students as opposed to typical hearing screening personnel like school health nurses (although this may differ across countries) conducted the screening. The hearScreen ${ }^{\mathrm{TM}}$ interface is however designed with preprogrammed protocols aimed at minimizing outcome variability between different testers. Another limitation of the current study is that only pilot clinical field trial results are reported without
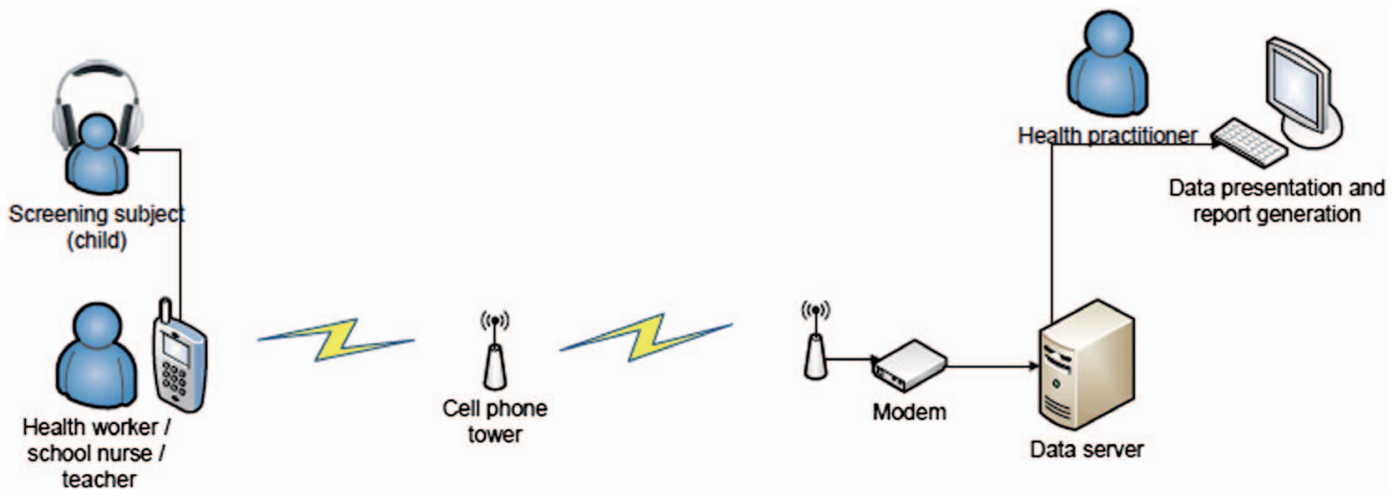

Figure 6. Illustration of hearing screening data sharing from the school site using mobile phone connectivity. 
diagnostic follow-up testing to ascertain true sensitivity and specificity rates. Field trials are however underway and we will report on these outcomes in due course. The test time is also a variable that may be different in test environments that have more ambient noise level interference. In the current study however the conventional and smartphone screening was conducted in the same environment in representative public schools of the City of Tshwane, South Africa. The fact that RETSPL levels for the Sennheiser HD202 headphones is not yet available is another limitation of the current study. Although also a supra-aural headphone like the TDH39 headphones, which served as the RETSPL calibration reference (ISO 389-1:1998; ANSI/ASA S3.6:2010), differences in acoustic impedance due to coupling differences are likely to result in slight variation to the RETSPL between headphones (Poulsen \& Oakley, 2009). As indicated however, RETSPLs are presently being established for the Sennheiser HD202 headphones according to ISO 389-9 (2009). Other factors that require further investigation include establishing the battery capacity of the smartphone for continuous screening. In our field trial experience, where the smartphones are typically used for five hours of screening, they accommodate this with ease without requiring to be recharged.

\section{Conclusions}

Low-cost solutions for hearing screening may make early detection more widely available as is proposed by current guidelines (AAA, 2011; ASHA, 1997; Skarzynski \& Piotrowska, 2012). This is especially important in developing areas of the world where more than $80 \%$ of persons with hearing loss reside without real prospects of early detection of hearing loss (WHO, 2013a; McPherson et al, 2010). As an example, a recent integrated school health policy in South Africa mandated annual hearing screening of all grade 1 pupils, totaling more than 1.1 million (IHSP, 2012). The most significant barriers to effective implementation of this hearing screening service, however, includes the high cost of existing screening audiometers, extensive training requirements for screeners in audiometric principles, overreferrals due to lack of environmental noise monitoring, and poor data capturing and management.

The hearScreen ${ }^{\mathrm{TM}}$ application was developed as an inexpensive alternative to conventional screening audiometry with specific applications to school-based screening. The application utilizes inexpensive, widely available, smartphone (US\$80) and headphone (US\$35) technology for hearing screening. Automated test sequences mitigate the subjective influence of screening personnel and minimizes training needs for screeners. Integrated real-time monitoring of environmental noise levels provides a measure of quality control whilst data capturing and subsequent sharing from the smartphone through cellular networks or WiFi streamline and simplify data management.

Findings from the validation study demonstrate that for hearing screening purposes (1) accurate acoustic calibration can be attained according to current standards (ANSI/ASA S3.6-2010; ISO 389-1, 1998); (2) valid monitoring of environmental noise can be done according to MPANLs and; (3) no significant difference for screening outcomes using smartphone hearScreen ${ }^{\mathrm{TM}}$ and conventional audiometry is evident.

\section{Acknowledgements}

The authors would like to acknowledge the audiology students at the University of Pretoria, who assisted in hearing testing, and the Tshwane school children who participated in this study.
Declaration of interest: The hearScreen ${ }^{\mathrm{TM}}$ application is intellectual property owned, patented and trademarked by the University of Pretoria. The product is being developed for commercialization and more information is available at www.hearscreen.co.za

\section{References}

American Academy of Audiology (AAA) 2011. American Academy of Audiology Clinical Practice Guidelines Childhood Hearing Screening. Available from: http://www.audiology.org/resources/documentlibrary/ Documents/ChildhoodScreeningGuidelines.pdf.

American National Standards Institute / Acoustical Society America (ANSI/ ASA) S3.1-1999 (R2013). Maximum Permissible Ambient Noise Levels for Audiometric Test Rooms.

American National Standards Institute / Acoustical Society America (ANSI/ ASA) S3.6-2010. Specification for Audiometers.

American Speech-Language Hearing Association 1997. Guidelines for audiologic screening. ASHA, 1-63.

ANSI (1983). ANSI S1.4-1983, Specification for Sound Level Meters (American National Standards Institute, New York) [1983 (R2007)].

Bamford J., Fortnum H., Bristow K., Smith J., Vamvakas G. et al. 2007. Current practice, accuracy, effectiveness, and cost-effectiveness of the school-entry hearing screening. Health Technol Assess, 11, 1-168.

Bess F., Dodd-Murphy J. \& Parker R. 1998. Minimal hearing loss in children: Prevalence, educational progress and functional status. Ear Hear, 19, 339-354.

Clark J.L. \& Swanepoel D.W. 2014. Technology for hearing loss: As we know it and as we dream it. Disabil Rehabil Assist Technol (Epub ahead of print).

Fagan J.J. \& Jacobs M. 2009. Survey of ENT services in Africa: Need for a comprehensive intervention. Global Health Action, 1-7.

FitzZaland R. \& Zink G. 1984. A comparative study of hearing screening procedures. Ear Hear, 5, 205-210.

Foulad A., Bui P. \& Djalilian H. 2013. Automated audiometry using apple iOS-based application technology. Otolaryngol Head Neck Surg, 700-706.

Goulios H. \& Patuzzi R.B. 2008. Audiology education and practice from an international perspective. Int J Audiol, 47, 647-664.

Handzel O., Ben-Ari O., Damian D., Priel M.M., Cohen J. et al. 2013. Smartphone-based hearing test as an aid in the initial evaluation of unilateral sudden sensorineural hearing loss. Audiol Neurotol, 18, 201-207.

Holden-Pitt L. \& Diaz J. 1998. Thirty years of the annual survey of deaf and hard of hearing children and youth: A glance over the decades. Am Ann Deaf, 143, 72-76.

Integrated School Health Policy (ISHP) 2012. Department of Basic Education and Department of Health. Pretoria, South Africa. Available from: http://0-www.doh.gov.za.innopac.up.ac.za/docs/policy/2012/ Integrated_School_Health_Policy.pdf.

International Electrotechnical Commission (IEC) 60645-1 (2012). Electroacoustics - Audiological equipment. Part 1 - Equipment for puretone audiometry.

International Standardization Organization (ISO) 389-1 (1998). Acoustics - Reference zero for the calibration of audiometric equipment. Part 1: Reference equivalent threshold sound pressure levels for pure tones and supra-aural earphones.

International Standardization Organization (ISO) 389-8 (2004). Acoustics - Reference zero for the calibration of audiometric equipment. Part 8: Reference equivalent threshold sound pressure levels for pure tones and circumaural earphones.

International Standardization Organization (ISO) 389-9 (2009). Acoustics - Reference zero for the calibration of audiometric equipment. Part 9: Preferred test conditions for the determination of reference hearing threshold levels.

Internet World Stats. 2014. Usage and Population Statistics. Available from: http://www.internetworldstats.com/stats.htm [26 February 2014]. 
Kam A.C.S., Gao H., Li L.K.H., Zhao H., Qiu S. et al. 2013. Automated hearing screening for children: A pilot study in China. Int J Audiol, 52, 855-860.

Kam A.C.S., Sung J.K.K., Lee K., Wong T.K.C. \& Van Hasselt A. 2012. Clinical evaluation of a computerized self-administered hearing test. Int J Audiol, 51, 606-610.

Kardous C.A. \& Shaw P.B. 2014. Evaluation of smartphone sound measurement applications. JASA Express Letters, 135, EL186-192.

Kelly T. \& Minges M. 2012. Exclusive Summary. Washington: World Bank.

Khoza-Shangase K. \& Kassner L. 2013. Automated screening audiometry in the digital age: Exploring uhear and its use in a resource-stricken developing country. Int J Technol Assess Health Care, 29, 42-47.

Lo A.H. \& McPherson B. 2013. Hearing screening for school children: Utility of noise-cancelling headphones. BMC Ear Nose Throat Disord, $12,6$.

McKay S., Gravel J. \& Tharpe A. 2008. Amplification considerations for children with minimal or mild bilateral hearing loss or unilateral hearing loss. Trends Amplif, 12, 43-54.

McPherson B., Law M.M. \& Wong M.S. 2010. Hearing screening for school children: Comparison of low-cost, computer-based and conventional audiometry. Child Care Health Dev, 36, 323-331.

Poulsen T. \& Oakley S. 2009. Equivalent threshold sound pressure levels (ETSPL) for Sennheiser HDA 280 supra-aural audiometric earphones in the frequency range 125 to $8000 \mathrm{~Hz}$. Int J Audiol, 48, 271-276.

Skarzynski H. \& Piotrowska A. 2012. Screening for pre-school and school-age hearing problems: European consensus statement. Int J Pediatr Otorhinolaryngol, 76, 120-121.
Swanepoel D., Clark J.L., Koekemoer D., Hall J.W., Krumm M. et al. 2010a. Telehealth in audiology: The need and potential to research underserved communities. Int J Audiol, 49, 195-202.

Swanepoel D. \& Hall J.W. 2010. A systematic review of telehealth applications in audiology. Telemed $J$ e-Health, 16, 181-200.

Swanepoel D., Mngemane S., Molemong S., Mkwanazi H. \& Tutshini S. 2010b. Hearing assessment: Reliability, accuracy and efficiency of automated audiometry. Telemed $\mathrm{J}$ e-Health, 16, 557-563.

Szudek J., Ostevik A., Dziegielewski P., Robinson-Anagor J., Gomaa N. et al. 2012. Can u hear me? Validation of an iPod-based hearing loss screening test. J Otolaryngol Head Neck Surg, 41, S78-S84.

Tharpe A. \& Bess F. 1991. Identification and management of children with minimal hearing loss. Int J Pediatr Otorhinolaryngol, 21, 41-50.

World Health Organization. 2013a. Millions of People in the World have a Hearing Loss that can be Treated or Prevented. Geneva: Switzerland. Available from: http://0-www.who.int.innopac.up.ac.za/pbd/deafness/ news/Millionslivewithhearingloss.pdf?ua $=1$ [30 January 2014].

World Health Organization. 2013b. Multi-country Assessment of National Capacity to Provide Hearing Care. Geneva: Switzerland. Available from: http://0-www.who.int.innopac.up.ac.za/pbd/publications/ WHOReportHearingCare_Englishweb.pdf. [6 March 2014].

World Health Organization. 2010. Neonatal and infant hearing screening. Current issues and guiding principles for action. Outcomes of a WHO informal consultation held at World Health Organization headquarters (9-10 November 2009), Geneva, Switzerland.

Wu W., Lu J., Li Y., Kam A.C.S., Tong M.C.F. et al. 2014. A new hearing screening system for preschool children. Int J Pediatr Otorhinolaryngol, 78, 290-295. 